den der unbestrahlten, nicht mit DNP versetzten Schnitte abgefallen war.

Die Versuche zeigen, daß die strahlenbedingte Atmungssteigerung bei etwa der gleichen Dosis einsetzt wie die strahlenbedingte Hemmung der durch DNP gesteigerten Atmung. Diese Beobachtung kann im Gegensatz zu unseren früheren Schlußfolgerungen nicht als Argument gegen eine entkoppelnde Wirkung der Be-

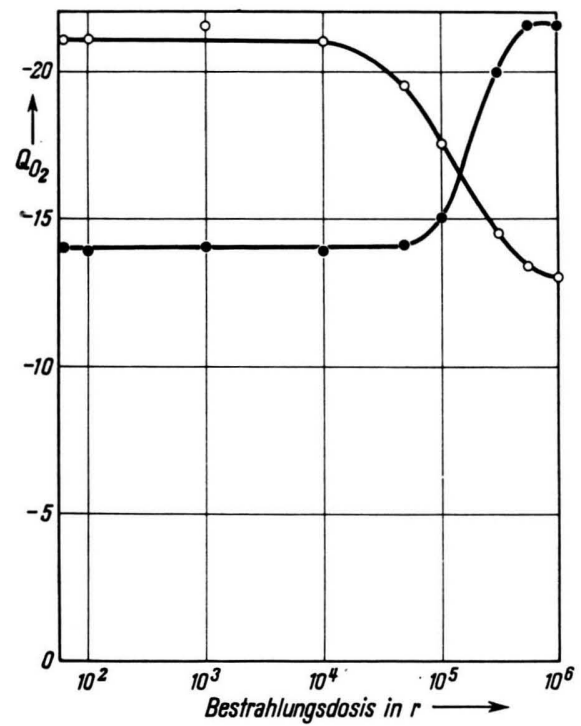

Abb. 1. Wirkung einer Bestrahlung auf die Atmung von Nierencortexschnitten (Meerschweinchen) ohne und mit Zusatz von 2.4-Dinitrophenol (Endkonzentration 0,2 mMol). Die Bestrahlung der Schnitte erfolgte bei $+1^{\circ}$ (vgl. l. c. $\left.{ }^{1}\right)$. Die in der Abb. 1 angegebenen Atmungsquotienten wurden während der 1. Stde. nach Bestrahlung ermittelt. —— : ohne Zusatz von DNP. $\bigcirc-O$ : mit Zusatz von DNP. strahlung gewertet werden. Es ist nämlich durchaus denkbar, daß die durch DNP bewirkte Atmungssteigerung, die ihrerseits eine Folge der Entkopplung der oxydativen Phosphorylierung ist, zusammen mit der strahlenbedingten Atmungssteigerung schließlich zu einer Hemmung der Sauerstoffaufnahme führt. Eine ähnliche Wirkung ist auch für DNP bekannt; hier schlägt die gesteigerte Gewebeatmung nach Erhöhung der DNP-Konzentration in eine Hemmung um, während die oxydative Phosphorylierung weiterhin entkoppelt bleibt ${ }^{3}$. Demnach könnte das Zusammentreffen der entkoppelnden Wirkung von DNP mit der möglichen entkoppelnden Wirkung der Bestrahlung durchaus eine Hemmung der Atmung bewirken. Die Tatsache, daß die strahlenbedingte Atmungssteigerung bei gleicher Dosishöhe wie die Hemmung der durch DNP gesteigerten Atmung eintritt, könnte für eine Summation des DNP. und des Bestrahlungseffektes im oben angedeuteten Sinne sprechen.

Eine endgültige Antwort auf die Frage, ob in dem hier untersuchten System die oxydative Phosphorylierung durch die Bestrahlung tatsächlich entkoppelt wird, kann jedoch auf Grund der beschriebenen Versuche noch nicht gegeben werden. Eine Aussage darüber ließe sich nur mit Hilfe der Bestimmung des P/O-Quotienten machen. Aus methodischen Gründen ist aber eine solche Bestimmung an Gewebeschnitten direkt nicht durchführbar. Die an isolierten Mitochondrien gemachten Beobachtungen hinsichtlich der Strahlenwirkung auf die oxydative Phosphorylierung ${ }^{5}$ lassen sich naturgemäß nicht auf die weitgehend intakten Zellen im Schnitt übertragen.

Die vorliegenden Untersuchungen wurden mit Unterstützung des $\mathbf{B}$ u $\mathbf{n}$. desministers für Atomkernenergie und Wasserwirt. $\mathrm{s}$ c h a f t durchgeführt.

5 B. Rajewsky, G. Gerber u. H. Pauly, Strahlentherapie 102, 517 [1957].

\section{Wirkungen von Insektiziden auf die Katalaseaktivität von Kresse-Keimlingen *}

Von Frank Beye

Im Botanischen Institut der Universität Freiburg i. Br.

(Z. Naturforschg. 15 b, 470-472 [1960]; eingegangen am 11. Februar 1960)

ZIEGLER $^{1}$ (1957) beobachtete an Heoleda-Sprossen eine Assimilations-Hemmung durch E 605 bei gleichzeitiger Steigerung der Atmung. Aus dieser Wirkung des Phosphorsäureesters ergab sich die Frage, welche stoffwechselphysiologischen Vorgänge bei der Wirkung beteiligt sein können. Da Katalasen einerseits bei der Kohlensäure-Assimilation, andererseits bei der Dissimilation regulierend eingreifen können (BERsin $\left.{ }^{2} 1954\right)$, wurde die Wirkung von verschiedenen Insektiziden auf

* Diese Untersuchungen wurden durch die De u t s che F orschungsgemeins chaf t ermöglicht, der für die Unterstützung gedankt sei.

1 H. Ziegler, Biol. Zbl. 76, 43 [1957].
Gartenkresse (Lepidium sativum L.) in diesem Sinne überprüft, nachdem bereits die Wachstumsbeeinflussung an Kressewurzeln untersucht worden war (BEYE, $1960 \mathrm{a}$ ).

Gut verlesene Samen ** wurden nach Wägung auf Torsionswaage in Petrischalen $(10 \mathrm{~cm} \phi)$ mit Rundfiltern (Schleicher \& Schüll 595) und $4 \mathrm{ml}$ dest. Wasser oder Testlösung im Brutschrank bei $26{ }^{\circ} \mathrm{C} 24$ oder $48 \mathrm{Stdn}$. kultiviert. Die Wägung erfolgte an den trockenen Samen, da die Keimpflänzchen sich nach Kultur schlecht quantitativ trocknen ließen und die gallertig gequollenen Samenschalen jeweils beim Zerreiben des Pflanzenmaterials mit verarbeitet wurden. Die Meßwerte wurden auf die Trockengewichte der Samen bezogen. Bei 24-Stdn.-Testen wurden jeweils 25, bei 48 Stdn. 10 Samen in jede Schale verbracht. Jede Testserie wurde wenigstens 5 -mal wiederholt (bei 5 Wieder-

2 T. Bersin, Kurzes Lehrbuch der Enzymologie. 4. Auflage. Akademie Verlag, Leipzig 1954.

3 F. BEYE, Z. Pflanzenkrankh. (Pflanzenpathol.) Pflanzenschutz [1960], im Druck.

** Saatgut: Fa. K. Hild, Marbach/Neckar. 
holungen also pro Konzentration eines Mittels wenigstens 15 Meßwerte). Die Katalaseaktivität wurde nach STE${ }_{\text {PHAN }}{ }^{4}$ (1932) bestimmt: $25 \mathrm{ml}$ Reibschalenhomogenate von Kressekeimlingen (Phosphatpuffer $m / 60, p_{\mathrm{H}} 7,0$ ) unter Einkippen von $10 \mathrm{ml} \mathrm{H}_{2} \mathrm{O}_{2}(1 \%)$ aus Innenbirne in den Reaktionsraum der Untersuchungsgefäße. Die Gefäße sind an Hg-Manometer angeschlossen und bei Schüttelung (etwa 100 pro Min.) werden nach 5 Min. die Volumänderungen bestimmt. Die Wirkungen der Insektizide sind als Differenz zu den Werten unbehan- delter Kontrollen angegeben (Weiteres zur Methode BEYE $^{5} 1960$ b). Die Kontrollwerte für 24 Stdn. ergaben eine mittlere Katalaseaktivität von $1,09 \mathrm{~mm} \mathrm{Hg}$, für $48 \mathrm{Stdn}$. eine solche von $6,74 \mathrm{~mm} \mathrm{Hg}$ pro Trockengewichtseinheit bei einem Zeitfehler zwischen Ansatz und Bestimmung von \pm 1 Stunde.

Folgende Insektizide wurden in wäßriger „Lösung“ der Handelspräparate im Konzentrationsbereich von $0,1-0,001 \%$ untersucht:

\begin{tabular}{|c|c|c|c|c|}
\hline \multicolumn{2}{|c|}{ Handelspräparat } & \multirow{2}{*}{\begin{tabular}{l}
\multicolumn{1}{c}{ Wirkstoff } \\
DDT \\
Phenkapton \\
Diazinon \\
Chlorbenzilat \\
Parathion \\
Metasystox
\end{tabular}} & \multirow{2}{*}{$\begin{array}{c}\begin{array}{c}\text { Wirkstoffgehalt } \\
{[\%]}\end{array} \\
50 \\
20 \\
20 \\
25 \\
50 \\
50\end{array}$} & \multirow{2}{*}{\begin{tabular}{l}
\multicolumn{1}{c}{ Hersteller } \\
Geigy, Basel \\
Geigy, Basel \\
Geigy, Basel \\
Geigy, Basel \\
Bayer, Leverkusen \\
Bayer, Leverkusen
\end{tabular}} \\
\hline $\begin{array}{l}\text { * Gesarol } 50 \\
\text { * Phenkapton } \\
\text { * Basudin } \\
\text { * G } 338 \\
\text { ** E } 605 \\
\text { ** Metasystox }\end{array}$ & $\begin{array}{l}\text { (Spritzp.) } \\
\text { (Spritzp.) } \\
\text { (Emulsion) } \\
\text { (Emulsion) } \\
\text { (Emulsion) } \\
\text { (Emulsion) }\end{array}$ & & & \\
\hline
\end{tabular}

* Angaben der J. R. Geigy A.G., Basel.

Alle untersuchten Präparate hemmten die Katalaseaktivität der Kresse. Nach 24-stdg. Einwirkung der Mittel auf die Samen ist die Abstufung der Katalasewirkung wie folgt:

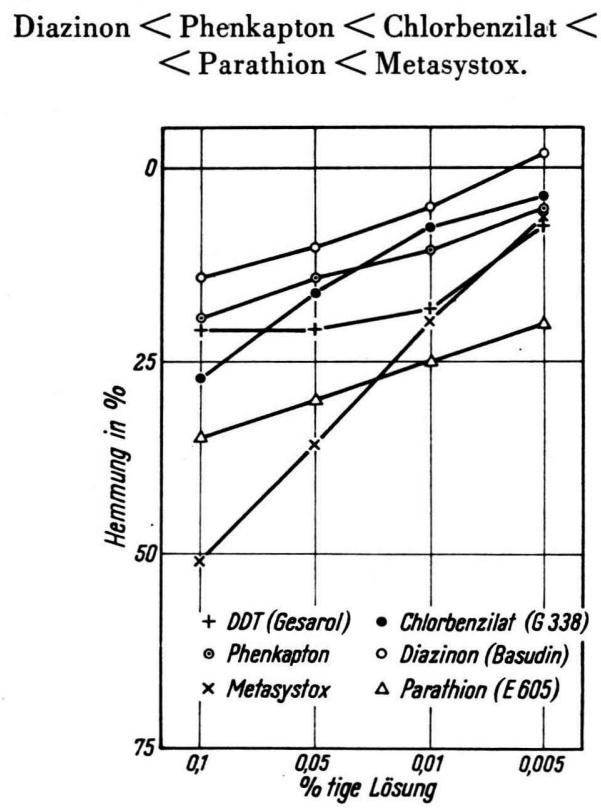

Abb. 1. Katalase-Hemmkurven nach 24 Stunden.

Die Katalasehemmung nimmt in der gegebenen Reihenfolge zu (Abb. 1). Die Hemmwerte sind für die erstgenannten 4 Mittel sehr ähnlich im Konzentrationsbereich von $0,1-0,05 \%$, geringere Konzentrationen $(0,01-0,005 \%)$ hemmen nicht sehr deutlich. Nach Parathion-Behandlung $(0,1-0,005 \%)$ nimmt die Hemmung weniger mit der Verdünnung ab (etwa 35 bis
** Nach Beran-Neururer (1955).

$20 \%)$. Metasystox hat bei $0,1-0,05 \%$ eine noch stärkere Hemmung der Katalaseaktivität zur Folge, aber bei $0,01-0,005 \%$ praktisch gleich geringe Wirkung wie die erstgenannten Mittel.

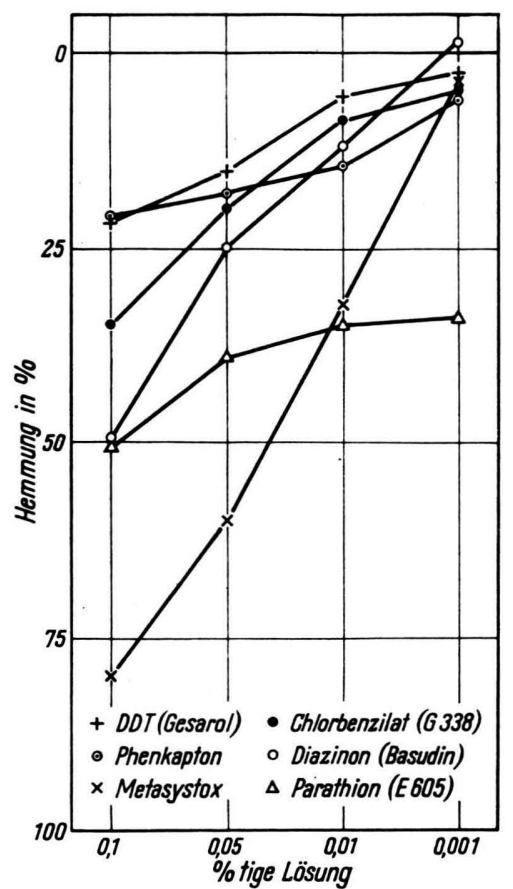

Abb. 2. Katalase-Hemmkurven nach 48 Stunden.

Nach 48 Stdn. Einwirkung ließe sich die abgestufte Wirkung in folgender Reihenfolge vom geringsten zum stärksten Eingriff darstellen:

4 J. Stephan, Jb. wiss. Bot. 75, 771 [1932]. 


\section{DDT $<$ Phenkapton $<$ Chlorbenzilat $<$ Diazinon $<$ Parathion $<$ Metasystox.}

Vergleicht man die Hemmkurven nach $48 \mathrm{Stdn}$. (Abb. 2) mit dem Kurvenbild nach 24 Stdn. (Abb. 1), so beabachtet man bei allen Mitteln, ausgenommen dem DDT-Präparat eine Zunahme der Hemmung. Diese verstärkte Abnahme der Katalaseaktivität ist bei Phenkapton und Chlorbenzilat gering, deutlicher beim Diazinon, am stärksten bei Metasystox und Parathion. Wie beim 24-Stdn.-Test ist die Wirkung des Parathions weniger deutlich konzentrationsabhängig abgestuft $(50$ bis 35\% Hemmung) als bei den anderen Mitteln.

Das Ausplanimetrieren des gesamten gezeichneten Hemmungsbereiches der Mittel in Abb. 1 und 2 ergab die Werte der Tab. 1, die die Ergebnisse in vereinfachten Zahlenwerten darstellen. Die Betrachtungsweise berücksichtigt mehr den gesamten Hemmbereich und weniger die konzentrationsabhängigen Unterschiede, bestätigt jedoch die oben gegebenen Abstufungen der Mittelwirkung nach 24- und 48-stdg. Einwirken. Berücksichtigt man aber die unterschiedlichen Wirkstoffgehalte der Mittel, so wäre die Wirkung auf die Katalaseaktivität der Kressekeimlinge nach 24 Stdn. gleich bei DDT und Diazinon. Chlorbenzilat, Phenkapton, Parathion und Metasystox haben untereinander fast gleiche, den beiden ersten Mitteln gegenüber aber sehr viel stärkere Wirkung nach 24 Stdn. zur Folge. Nach 48 Stdn. ist bei Berücksichtigung des Wirkstoffgehaltes die Wirkung wie folgt abgestuft :

$$
\begin{gathered}
\text { DDT }<\text { Chlorbenzilat } \\
=\text { Phenkapton }<\text { Parathion }<\text { Diazinon }=\text { Metasystox } .
\end{gathered}
$$

Hiernach ergibt sich neben den Unterschieden nach 24 und $48 \mathrm{Stdn}$. die besonders auffällige Verstärkung der Wirkung des Diazinonpräparates nach $48 \mathrm{Stdn}$., die sich in einer um das Doppelte zunehmenden Hemmung äußert. Diese Umrechnung der Mittelwirkung kann jedoch nicht ohne weiteres allein auf den Wirkstoffgehalt der Präparate bezogen werden, da die Mittel in verschiedenen Formulierungen vorliegen (Spritzpulver und Emulsionen) und diese wiederum in ihrer Zusammensetzung im einzelnen unterschieden sind. Die in den Präparaten enthaltenen Lösungshilfsmittel und Bei-

\begin{tabular}{|c|c|c|}
\hline \multirow[t]{2}{*}{ Wirkstoff (Präparat) } & \multicolumn{2}{|c|}{$\begin{array}{c}\text { Planimetrische Werte } \\
\text { der Katalasehemmkurve }\end{array}$} \\
\hline & 24 Stdn. & 48 Stdn. \\
\hline DDT (Gesarol 50) & 32,5 & 19,8 \\
\hline Chlorbenzilat (G 338) & 23,7 & 29,0 \\
\hline Phenkapton & 22,8 & 27,6 \\
\hline Diazinon (Basudin) & 13,5 & 36,6 \\
\hline Parathion (E 605) & 49,6 & 70,7 \\
\hline Metasystox & 50,9 & 80,6 \\
\hline
\end{tabular}
stoffe können Eindringen und Wirkung im Pflanzen-
Tab. 1. Planimetrie der Hemmkurven von Abb. 1 und 2. (Flächenplanimetrie, die Werte geben die Abweichungen der Kurven gegenüber den jeweiligen Kontrollen an.)

gewebe unterschiedlich beeinflussen, so daß hier nicht ohne weiteres allein auf die pflanzenphysiologische Wirkung der Wirksubstanzen geschlossen werden kann.

Bei den verhältnismäßig ähnlich verlaufenden Hemmkurven der Mittel ist ein zusammenfassender Vergleich, wie er auf Grund der Tab. 1 angestellt wurde möglich, wenn man von der Parathion-Wirkung absieht. Das E 605-Präparat nimmt eine Sonderstellung ein: Bei 24 sowohl wie $48 \mathrm{Stdn}$. hat es die am stärksten anhaltende Wirkung und ist bei $0,001 \%$, also Lösungen, die etwa dem Bereiche der in der Praxis geübten Spray. behandlung entsprechen, mit $20-35 \%$ Hemmung das am stärksten in schwächeren Konzentrationen eingreifende Mittel. Diese Wirkung des Parathions auf die Katalaseaktivität ist nach unseren Beobachtungen sehr ähnlich den Wachstumshemmungen bei Kressekeimlingen. Das Wurzelwachstum wurde durch E 605 ähnlich anhaltend in geringeren Konzentration gehemmt, während bei den anderen Insektiziden die Wirkungen schneller abklangen (BEYE ${ }^{3} 1960 \mathrm{a}$ ).

Es stellt sich die Frage, ob die Wirkung der Präparate auf die Katalaseaktivität der Kressekeimlinge mit der unterschiedlichen Beeinflussung von Atmung und Assimilation wie beim Parathion (ZIEGLER ${ }^{1}$ 1957) verkettet sind. Unsere Untersuchungen über die Beeinflussung der Atmung an Kressekeimlingen erlauben hier noch keine Stellungnahme.

Die Vergleiche werden mit anderen Mitteln und anderen Versuchspflanzen fortgesetzt.

5 F. BeYe, Z. Flora [1960], im Druck. 